University of South Carolina

Scholar Commons

$12-2006$

\title{
A Novel Account of Scientific Anomaly: Help for the Dispute over Low-Dose Biochemical Effects
}

Kevin C. Elliott

University of South Carolina - Columbia, elliotkc@mailbox.sc.edu

Follow this and additional works at: https://scholarcommons.sc.edu/phil_facpub

Part of the Philosophy Commons

\section{Recommended Citation}

(C) 2006 by the Philosophy of Science Association http://www.philsci.org/

This Article is brought to you by the Philosophy, Department of at Scholar Commons. It has been accepted for inclusion in Faculty Publications by an authorized administrator of Scholar Commons. For more information, please contact digres@mailbox.sc.edu. 


\title{
A Novel Account of Scientific Anomaly: Help for the Dispute over Low-Dose Biochemical Effects
}

\author{
Kevin C. Elliott $\$$
}

The biological effects of low doses of toxic and carcinogenic chemicals are currently a matter of significant scientific controversy. This paper argues that philosophers of science can contribute to alleviating this controversy by examining it with the aid of a novel account of scientific anomaly. Specifically, analysis of contemporary research on chemical hormesis (i.e., alleged beneficial biological effects produced by low doses of substances that are harmful at higher doses) suggests that scientists may initially describe anomalous phenomena in terms of multiple distinct "characterizations," each of which is compatible with current empirical evidence. By focusing attention on this feature of scientific anomalies, philosophers of science can alleviate the controversy over low-dose chemical effects in at least two ways: (1) they can pinpoint the significant ways in which particular characterizations frame the controversy, and (2) they can identify the methodological value judgments at stake in researchers' choice of characterizations.

1. Introduction. Scientific anomalies are of significant theoretical interest in the philosophy of science, and they also play an important practical role in some biological controversies. In the philosophy of science, anomalies are important for at least two related reasons. First, most of the classic twentieth-century works on scientific theory change, including those of Karl Popper (1959), Thomas Kuhn (1970), Imre Lakatos (1970), and Larry Laudan (1977), suggested that anomalies play a crucial "catalytic" role in the processes by which scientists develop new theories and alter previous ones. Second, many recent studies argue that the process of scientific discovery often involves a gradual modification and elaboration of existing theories or models in response to scientific problems,

$\dagger$ To contact the author write to: Department of Philosophy, University of South Carolina, Columbia, SC 29208; email: ke@sc.edu.

$\ddagger I$ would like to thank Kristin Shrader-Frechette for very helpful input on earlier versions of this paper. 
many of which involve anomalies (Wimsatt 1987; Darden 1991; Schaffner 1993; Nickles 1997; Elliott 2004a). Thus, both classic discussions of theory change and contemporary studies of scientific discovery affirm that anomalies are central to scientific progress.

Some of the literature on science policy suggests that anomalies also have the potential to play an important role in many scientific controversies, especially those with practical ramifications. For example, when a body of scientific evidence supports a particular policy that runs counter to a political actor's own agenda, he or she may search for and emphasize anomalous scientific data as a way of resisting the evidence for the policy (Herrick and Sarewitz 2000; Sarewitz 2000). Furthermore, whereas anomalies in highly theoretical areas of science might "simmer" relatively unnoticed for an extended period of time, thus enabling researchers to develop increased understanding of them (Kuhn 1970), the policy process is likely to bring anomalies to the political front burner immediately, when scientists still know very little about their characteristics (Collins and Evans 2002).

Section 2 of this paper examines contemporary controversies concerning the low-dose biological effects of toxic chemicals and argues that anomalies do indeed play a central role in these disputes. On the basis of this case study, Section 3 suggests a novel philosophical account of scientific anomaly that emphasizes the potential for anomalies to display multiple "characterizations." Section 4 of the paper argues that this account suggests at least two ways in which philosophers of science can help to resolve the biological controversy over low-dose chemical effects.

2. The Biological Controversy over Low-Dose Chemical Effects. The lowdose biological effects of toxic and carcinogenic chemicals are currently a matter of hot policy debate. On one hand, researchers such as Theo Colborn claim that extremely low doses of many chemicals may mimic hormones such as estrogen and be responsible for dramatic declines in animal populations. Some claim that these "endocrine-disrupting" chemicals may also be related to human reproductive cancers, immune disorders, and declining male sperm counts (Colborn, Dumanoski, and Myers 1996). Nicholas Ashford adds that approximately 5\% of the U.S. population may suffer from extreme sensitivity to toxic chemicals. This phenomenon, which is frequently called "multiple chemical sensitivity" (MCS), may be linked to "Gulf War syndrome," "sick-building syndrome," and other environmental sensitivities (Ashford and Miller 1998). On the other hand, the influential toxicologist Edward Calabrese suggests that low doses of many toxins may actually have beneficial effects. He claims that these beneficial effects, which he calls "chemical hormesis," are widely generalizable across different species, biological endpoints, and 
toxins, and he notes that this phenomenon "is counter to the cancer risk assessment practices by U.S. regulatory agencies . . . which assume that cancer risk is linear in the low-dose area" (Calabrese and Baldwin 1998, VIII-1; see also Calabrese and Baldwin 2003).

Beneficial effects produced by low doses of toxic chemicals are plausibly an example of a scientific anomaly. In order to predict the effects of toxins and carcinogens, toxicologists currently use models that predict either harmful effects at all dose levels or thresholds below which chemicals have no biological effect (Calabrese and Baldwin 2003). Although researchers and policy makers recognize that these models provide only rough approximations of actual chemical effects, the models are part of a toxicological framework in which toxic chemicals are expected to produce harmful effects (if they produce effects at all) at low doses. Thus, the occurrence of chemical hormesis is unexpected, or anomalous, relative to current toxicological science. Many of the phenomena associated with endocrine disruption and MCS are also anomalous (see Elliott 2004b). The rest of this paper focuses on hormesis as one particular example of anomaly in the controversy over low-dose chemical effects, but many features of the hormesis case also apply to the phenomena of MCS and endocrine disruption.

The effects of alcohol on human mortality provide a good illustration of the characteristics of the hormesis phenomenon (although not all researchers would consider the alcohol case as a genuine instance of hormesis). At high doses, alchohol increases human mortality, but at lower doses it can actually decrease the mortality rate below the level of controls (Gordon and Doyle 1987). The result is a U-shaped dose-response curve that reflects the production of opposite effects at low doses versus at high doses (see Figure 1), which is characteristic of hormetic responses to toxic chemicals. These sorts of hormetic effects can allegedly occur on endpoints such as fertility, cancer incidence, growth, body weight, or enzyme activity, in addition to mortality.

A particularly striking feature of the hormesis anomaly is that current researchers appear to be describing or "characterizing" it in multiple, different ways. Each of these characterizations involves an "empirical component" and a "theoretical component" that stand in a problematic relation to one another, but the components vary from one characterization to another. For example, when some researchers describe the hormesis anomaly, they focus on one or more sets of experimental data that are unexpected (e.g., Vichi and Tritton 1989; Davis and Svendsgaard 1990). If one were to make these researchers' characterizations of the anomaly as explicit as possible, they would seem to involve an inconsistency relation between statements that describe a set of data from one or 


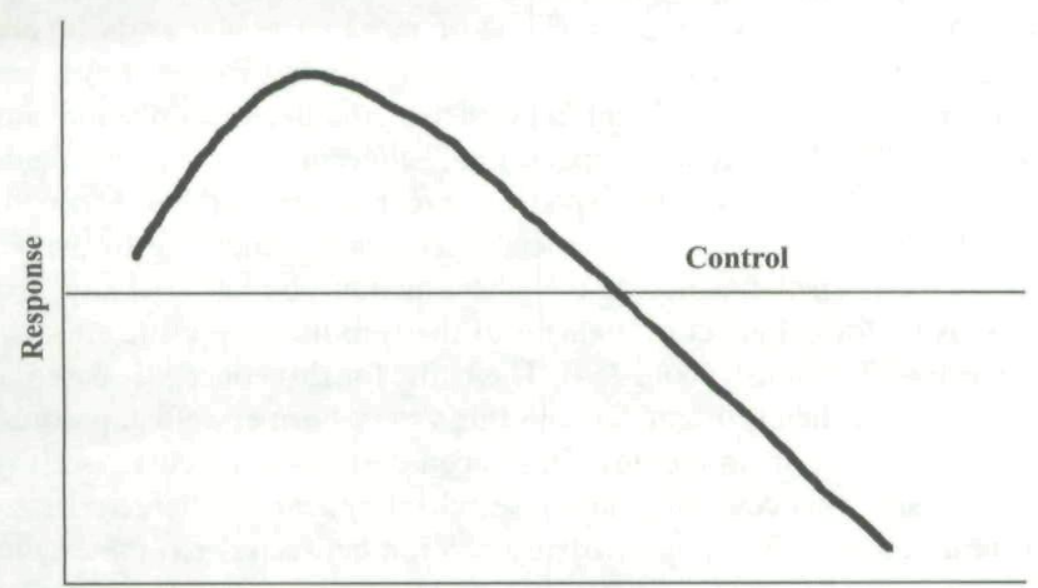

Dose

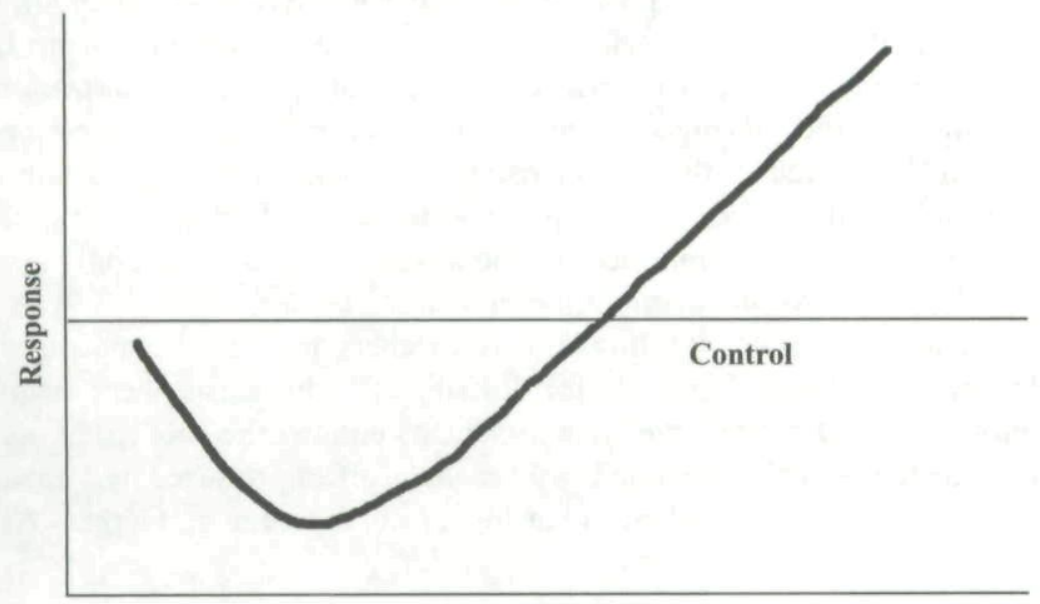

Dose

Figure 1. Examples of the general form of hormetic dose-response relationships. The bottom curve could represent the relationship between alcohol intake and human mortality, whereas the top curve could represent a hormetic relationship between dose of a growth inhibitor and plant growth. 
more experiments on one hand and statements that describe researchers' expectations for those data on the other hand.

Other researchers appear to conceptualize the anomalous research data as evidence for the occurrence of one or more particular sorts of phenomena. ${ }^{1}$ I have argued, however, that current researchers have been working with multiple different concepts for the hormesis phenomenon (Elliott 2000b). Two broadly "operational" concepts for the phenomenon include (1) "U-shaped-dose-response-curve hormesis" (henceforth "Ushaped hormesis") and (2) "beneficial hormesis."2 According to the first concept (U-shaped hormesis), the phenomenon consists of "any nonspurious biological effect of a chemical that produces opposite effects at higher doses" (Elliott 2000b, 354). The name for this concept is based on the fact that a chemical that displays this sort of hormetic effect produces either an upright or upside-down $\mathrm{U}$-shaped dose-response curve (see Figure 1). Using this concept, some researchers appear to characterize the hormesis anomaly as an inconsistency relation between descriptions of the occurrence of U-shaped hormesis on one hand and descriptions of doseresponse models that do not incorporate U-shaped responses on the other hand (see Calabrese and Baldwin 1998; Davis and Svendsgaard 1990). It is notable, however, that even researchers who agree on employing $U$ shaped hormesis as the empirical component of their characterizations may still vary their theoretical components. For example, Calabrese and Baldwin (1998) frequently characterize the hormesis anomaly as a failure of physiological theories to explain the occurrence of $U$-shaped hormesis. Thus, the theoretical component of the anomaly could be not only a set of models but also one or more physiological theories.

As I argued previously, however, researchers use other concepts, in addition to U-shaped hormesis, for describing the hormesis phenomenon (Elliott 2000b). For example, many scientists employ the concept of beneficial hormesis, which I defined as a beneficial effect produced by a chemical that produces harmful effects at higher doses (see, e.g., Gerber, Wil-

1. The term 'phenomenon' is used here to refer to a generalizable event that can be predicted by a theory and for which the data in particular experiments provide evidence (see Bogen and Woodward 1988; Woodward 2000).

2. For the purposes of this paper, an operational concept can be defined as one that is defined in terms of its criteria of application, which in the case of hormesis involves the measurement of some biological endpoint. A mechanistic concept involves the isolation of a system in which hormetic phenomena are produced by the interaction of parts according to causal laws (see, e.g., Bridgman 1927; Machamer, Darden, and Craver 2000). 
liams, and Gray 1999). ${ }^{3}$ Other researchers appear to define the hormesis phenomenon not merely in terms of particular operational effects but rather in terms of the mechanisms by which a chemical produces those effects. For example, Stebbing (1998) employs the concept of "overcompensation hormesis," which consists of opposite effects produced by "a biological response in which processes are stimulated to above-normal levels in an attempt to restore organismal homeostasis after it is altered by a toxic chemical" (Elliott 2000b, 356). ${ }^{4}$ Davis and Svendsgaard (1990) and other scientists have also hinted at the concept of "multiple-effects hormesis," which I defined as "a low-dose effect, opposite to that which occurs at higher doses, that is caused by multiple biological effects of a chemical influencing the same endpoint in different ways at different dose levels" (Elliott 2000b, 360). Researchers who employ these different concepts for the hormesis anomaly appear to be characterizing it in subtly different ways.

There even appear to be some characterizations of hormesis according to which the theoretical component of the anomaly is not merely a model or theory but an overarching paradigm. Especially in the early twentieth century, some researchers suggested that hormesis might be the same sort of phenomenon as homeopathy, even though they were not sure precisely how to characterize homeopathy itself (Calabrese and Baldwin 2000). Homeopathy is a debated medical practice in which people are treated for an illness by receiving extremely small quantities of substances that produce the same symptoms as the illness. Some homeopathic physicians make the surprising claim that their remedies are helpful even when they are diluted to the point that not even one molecule of the homeopathic substance is left in solution. Thus, at least some forms of homeopathic medicine run counter to paradigmatic assumptions of modern chemistry and physics, which hold that solutions do not retain the properties of substances that are no longer present in them. According to this characterization of the hormesis anomaly, it would consist of an inconsistency relation between descriptions of the phenomenon of hormesis (i.e., homeopathy) and descriptions of the current paradigms of chemistry, physics, and toxicology, which run counter to homeopathic phenomena.

3. The concept of beneficial hormesis is very similar to the concept of $U$-shaped hormesis, but the beneficial hormesis concept requires that the low-dose effect of a hormetic toxin must not merely be the opposite of what is produced at higher doses but must also be a beneficial effect.

4. Even though overcompensation hormesis does not involve one particular mechanism, it abstractly identifies a family of related mechanisms, so one might call it a mechanism "sketch" or "schema" (see Machamer et al. 2000). 
3. A Novel Account of Anomaly. This section argues that the hormesis case study suggests a novel account that emphasizes the multiplicity of characterizations with which researchers may describe an anomaly. The case illustrates two particularly important ways in which the characterizations of an anomaly may differ from one another. First, the "general type" of the empirical or theoretical components may vary. This paper defines the general types of anomaly components as broad categories that describe the sorts of entities that can serve as empirical or theoretical components. Sets of data and phenomena are two general types of empirical anomaly components in the hormesis case, whereas researchers' expectations, models, theories, mechanism schemata, and paradigms are general types of theoretical anomaly components. ${ }^{5}$ The characterizations of an anomaly may also vary in a second way if their components differ in what this paper will call their "specific type." The specific type is a particular instantiation of the general type; it can serve as the empirical or theoretical component in an actual characterization of an anomaly. For example, two characterizations of hormesis may both have the same general type of empirical component (namely, a phenomenon), but the specific type of empirical component may be the phenomenon of $\mathrm{U}$-shaped hormesis in one characterization and the phenomenon of beneficial hormesis in the other.

Previous accounts of anomaly do not appear to have placed major emphasis on either of these ways in which the characterizations of an anomaly can vary. First, they each focused attention on one or two particular general types of empirical and theoretical components (e.g., phenomena, theories, paradigms, or research programs) that anomalies sometimes display (see Popper 1959; Kuhn 1970; Lakatos 1970; Laudan 1977; Darden 1991). Second, they did not emphasize the ways in which the specific type of an anomaly's components could vary from one characterization to another; instead, they tended to describe an anomaly in terms of a specific phenomenon that conflicted with a particular theory or paradigm. More than other thinkers, Kuhn (1970) did draw some attention to the potential for an anomaly to be conceptualized differently as researchers converted from one paradigm to another, but his discussion of this feature of anomaly was not systematic. ${ }^{6}$

5. I thank Lindley Darden for suggesting that mechanism schemata may often serve as the theoretical component of anomalies in the biological sciences.

6. Although Darden has not previously emphasized how the general and specific types of an anomaly's components vary from one characterization to another, the process of developing multiple characterizations for an anomaly could plausibly be associated with her first two strategies or stages for anomaly resolution (i.e., confirming that an anomaly exists and localizing the problem) (Darden 1991). I have previously argued 
As a first step toward developing a novel account that emphasizes how anomalies may display multiple characterizations, this paper offers the following description of anomaly. It is called "account P" because it emphasizes the plurality of ways in which researchers may describe and conceptualize an anomaly.

Account P. A scientific anomaly is described by a "characterization," which consists of an empirical component, a theoretical component, and a problematic relation between them. An anomaly may admit of multiple characterizations that differ in the general and specific types of their components and the relation between them: ${ }^{7}$

(i) individual researchers may employ more than one characterization at roughly the same time,

(ii) different researchers may employ different characterizations at roughly the same time, and

(iii) researchers may change their characterizations over time.

In the hormesis case, for example, Calabrese and Baldwin's report on their initial literature study constitutes an example of how individual researchers can pursue more than one characterization at a time, because they employed some characterizations involving the phenomenon of $\mathrm{U}$ shaped hormesis and others using that of overcompensation hormesis (Calabrese and Baldwin 1998). Meanwhile, other researchers have emphasized different characterizations that do not conceptualize hormesis as a particular phenomenon but rather describe it in terms of experimental data that conflict with researchers' expectations for their experiments (e.g., Jonas 2001). Finally, Calabrese and Baldwin continue to alter their characterizations. After their initial literature study, they began to focus on "mechanistic" characterizations of hormesis as an overcompensation phenomenon (Calabrese 1999), but more recently they reverted to "operational" characterizations of the anomaly (Calabrese and Baldwin 2002).

In addition to the support that account $\mathrm{P}$ receives from the hormesis case (and the cases of MCS and endocrine disruption, as described in Elliott [2004b]), recent theoretical work in the philosophy of science also indicates the value of this description of anomaly. In particular, a number of thinkers have emphasized that experimental data often must be inter-

that these early stages need to be examined further and that they may play an important role in the process of scientific discovery (Elliott 2004a).

7. Note that even though this paper does not explore variations in the problematic relation that holds between the components of different anomaly characterizations, account $P$ suggests that the anomalous relation could also vary (including, e.g., relations of inconsistency, inexplicability, incompatibility, lack of conformation, and lack of homomorphism or isomorphism). 
preted extensively before they can be brought to bear in the justification or testing of theories (see Hacking 1988; Mayo 1996). For example, Bogen and Woodward (1988) argue that low-level experimental results are not directly explained by scientific theories; rather, researchers use experimental data to infer the occurrence of more generalizable phenomena. In addition, David Gooding (1990) has emphasized that, as scientists develop concepts for describing novel phenomena, they may start with intermediate descriptions of their perceptual experiences that he calls "construals." He suggests that researchers conceptualize their results only after a period of what might be called "exploratory experimentation" (Steinle 1997). These claims support the notion in account $P$ that the general or specific type of an anomaly's components may vary as researchers consider how to interpret their results.

4. How Account P Can Alleviate Controversy. By providing a more detailed analysis of the early stages in which scientists analyze anomalous findings, account $\mathrm{P}$ may suggest new insights concerning a variety of philosophical topics, including scientific discovery and the role of values in scientific practice (Elliott 2004b). This paper argues, in particular, that account $\mathrm{P}$ may help to alleviate biological controversies that involve anomalous findings. By highlighting the variety of characterizations that policy-relevant anomalies may display, this description of anomaly can contribute at least two sorts of insights. First, account P encourages philosophers of science to look for ways in which the characterizations of an anomaly play an important role in framing a controversy. Second, the account highlights the importance of identifying the methodological value judgments at stake in the choice of characterizations for anomalies that are implicated in biological controversies. By highlighting the framing effects and methodological value judgments associated with particular characterizations, philosophers of science can warn researchers and policy makers about anomaly conceptualizations that are particularly questionable or that have the potential to influence the ongoing controversy in significant ways.

4.1. Framing the Controversy. A number of different characterizations of hormesis have the potential to play an important role in framing the controversy concerning low-dose toxic chemical effects. First, characterizing the hormesis anomaly as a homeopathic phenomenon (that potentially conflicts with overarching paradigms of chemistry and physics) appears to decrease its legitimacy and to discourage research concerning it. For example, Calabrese suggests that the tendency for researchers to associate hormesis with homeopathy was a crucial factor contributing to the lack of attention that hormesis received from roughly the 1930 s to 
the 1980s (see Calabrese and Baldwin 2000). In contrast, the concepts of "overcompensation" or "multiple-effects" hormesis appear to increase its scientific legitimacy considerably. Each of these concepts characterizes hormesis in terms of specific, plausible mechanistic phenomena (either processes of overcompensation to toxins or multiple effects of a chemical on different biological endpoints). Thus, they make it appear more likely that hormesis is a legitimate phenomenon that deserves further research. Nevertheless, it is not clear that these concepts, by themselves, strongly support the significance of hormesis for public policy. For example, the concept of overcompensation hormesis suggests that toxic chemicals produce beneficial low-dose effects for only a short time (during which the overcompensation occurs), after which they could revert to neutral or harmful effects. The concept of multiple-effects hormesis indicates that hormetic effects may not be very generalizable from one chemical or endpoint to another, because those effects depend on the unique potential for particular chemicals to produce multiple effects on particular biological systems.

In contrast with other concepts of hormesis, that of beneficial hormesis does appear to draw attention to the potential policy significance of the phenomenon. It suggests that toxic chemicals may produce beneficial effects for organisms when administered at sufficiently low doses (which might seem to indicate that some regulations of toxic chemicals need to be relaxed). Nevertheless, this concept also has the potential to create confusion. It suggests that the new view about toxic chemicals is that they may be beneficial at low doses, in contrast to the old view (which is that they were harmful at low doses). But this dichotomy between old and new views may gloss over the fact that a toxic chemical can produce a beneficial effect on one endpoint over the short term while still having harmful effects on the organism as a whole over the long term. Thus, less normative concepts, such as $\mathrm{U}$-shaped hormesis, might be preferable to the concept of beneficial hormesis, because they might decrease the potential for people to assume that hormetic effects are beneficial for entire organisms over extended periods of time.

This possibility for the concept of beneficial hormesis to frame debates about hormesis in a manner that misleads the public is made even more serious because of the potential for researchers to conflate different concepts of the anomaly. For example, I have argued previously that Calabrese and Baldwin (1998) initially defended the generalizability of hormesis by providing evidence for the occurrence of $U$-shaped hormesis (Elliott 2000a). In the same publication (as well as in many other publications by them and other authors), however, they shifted to the concept of beneficial hormesis and suggested that their results encouraged reevaluation of government risk-assessment policy based on the likely 
occurrence of beneficial effects produced by low doses of toxic chemicals. They did not provide independent evidence, though, that opposite effects on individual endpoints over the short term are genuinely beneficial for the organism as a whole over the long term. Thus, Calabrese and Baldwin's conflation of different concepts may increase the potential for policy makers and members of the public to be confused about the likelihood that toxic chemicals are producing genuinely beneficial effects at low doses.

4.2. Methodological Value Judgments. Account $\mathrm{P}$ can also contribute to alleviating contemporary biological controversies over low-dose chemical effects by highlighting the methodological value judgments that are involved in choosing some characterizations of an anomaly rather than others. In the case of hormesis, two value judgments appear to be particularly important. The first has already been introduced by the preceding discussion of the beneficial hormesis concept and its framing effects. Researchers have to decide whether the evidence that $U$-shaped hormesis occurs provides adequate reason to think that beneficial hormesis also occurs. Gerber et al. acknowledge that apparent beneficial effects on one endpoint "might have the effect of altering an optimum resource allocation among such functions as growth, maintenance, and reproduction. . . . A higher level of performance in any one of these [as a result of a hormetic effect] might be achieved at the expense of the other two [thus resulting in negative effects for the organism as a whole]" $(1999,275)$. Nevertheless, they make the methodological value judgment that Calabrese and Baldwin have identified enough apparent instances of $U$-shaped hormesis on different endpoints to conclude that beneficial hormesis does occur (278). Other researchers, such as University of Missouri biologist Fred vom Saal, are very skeptical of the notion that $U$-shaped dose-response curves on particular endpoints are likely to result in benefical effects for an organism as a whole (see Kaiser 2003).

A second methodological judgment involves determining whether the literature-study approach employed by Calabrese and Baldwin (1998) provides sufficient evidence to conclude that the hormesis anomaly constitutes a widely generalizable phenomenon rather than an occasional set of anomalous data. On one hand, a number of researchers appear to think that Calabrese and Baldwin have uncovered very convincing evidence for a general phenomenon (e.g., Gerber et al. 1999). On the other hand, Jonas (2001) represents the views of another group of scientists when he claims that "Less than $1 \%$ of over 20,000 studies reviewed [by Calabrese and Baldwin] came close to true hypothesis testing of hormesis in experimental settings. . . . The criteria for rigorous 'proof' of hormesis will be different than those the authors [i.e., Calabrese and Baldwin] have used simply to 'identify' hormesis [sic] may exist. In the former one would want to assure 
proper dose verification, randomization of samples, blinding of outcome measures, proper statistical analysis, and full reporting of all data" (626627; see also Mayo and Spanos 2006).

5. Conclusion. This paper argued that attention to a novel account of scientific anomaly can contribute to the alleviation of biological controversies concerning the low-dose effects of toxic chemicals. It developed a new description of anomaly, called account $\mathrm{P}$, that emphasizes how researchers may employ multiple characterizations for a single anomaly. These characterizations involve empirical and theoretical anomaly components that vary in their "general" and "specific" types. The paper then suggested how, in the case of the hormesis anomaly, attention to this account can alleviate biological controversy. Account P encourages philosophers of science to identify the framing effects of particular characterizations and the methodological value judgments involved in employing some characterizations over others. By doing so, they can highlight anomaly conceptualizations that are likely to exacerbate or mitigate controversy.

\section{REFERENCES}

Ashford, Nicholas, and Claudia Miller (1998), Chemical Exposures: Low Levels and High Stakes. 2nd ed. New York: Wiley.

Bogen, J., and James Woodward (1988), "Saving the Phenomena," Philosophical Review 97: 303-352.

Bridgman, P. W. (1927), The Logic of Modern Physics. New York: Macmillan.

Calabrese, Edward (1999), "Evidence That Hormesis Represents an 'Overcompensation' Response to a Disruption in Homeostasis," Ecotoxicology and Environmental Safety 42: $135-137$.

Calabrese, Edward, and Linda Baldwin (1998), Chemical Hormesis: Scientific Foundations. College Station, TX: Institute for the Advancement of Chemical Technology.

- (2000), "Tales of Two Similar Hypotheses: The Rise and Fall of Chemical and Radiation Hormesis," Human and Experimental Toxicology 19: 85-97.

- (2002), "Defining Hormesis," Human and Experimental Toxicology 21: 91-97.

(2003), "Toxicology Rethinks Its Central Belief," Nature 421: 691-692.

Colborn, Theo, Diane Dumanoski, and J. P. Myers (1996), Our Stolen Future. New York: Dutton.

Collins, Harry, and R. Evans (2002), "The Third Wave of Science Studies: Studies of Expertise and Experience," Social Studies of Science 32: 235-296.

Darden, Lindley (1991), Theory Change in Science. New York: Oxford University Press.

Davis, J. Michael, and David Svendsgaard (1990), "U-Shaped Dose-Response Curves: Their Occurrence and Implications for Risk Assessment," Journal of Toxicology and Environmental Health 30: 71-83.

Elliott, Kevin (2000a), "A Case for Caution: An Evaluation of Calabrese and Baldwin's Studies of Chemical Hormesis," Risk: Health, Safety, and Environment 11: 177-196. - (2000b), "Conceptual Clarification and Policy-Related Science: The Case of Chemical Hormesis," Perspectives on Science 8: 346-366.

(2004a), "Error as Means to Discovery," Philosophy of Science 71: 1-24.

(2004b), Scientific Anomaly and Biological Effects of Low-Dose Chemicals. Ph.D. dissertation. South Bend, IN: University of Notre Dame.

Gerber, Linda M., George C. Williams, and Sandra J. Gray (1999), "The Nutrient-Toxin 
Dosage Continuum in Human Evolution and Modern Health," Quarterly Review of Biology 74: 273-289.

Gooding, David (1990), Experiment and the Making of Meaning: Human Agency in Scientific Observation and Experiment. Dordrecht: Kluwer.

Gordon, T., and J. Doyle (1987), "Drinking and Mortality: The Albany Study," American Journal of Epidemiology 125: 263-270.

Hacking, Ian (1988), "On the Stability of the Laboratory Sciences," Journal of Philosophy 85: 507-514.

Herrick, C., and Daniel Sarewitz (2000), "Ex Post Evaluation: A More Effective Role for Scientific Assessments in Environmental Policy," Science, Technology, and Human Values 25: 309-331.

Jonas, W. (2001), “A Critique of 'The Scientific Foundations of Hormesis,"” Critical Reviews in Toxicology 31: 625-629.

Kaiser, J. (2003), "Sipping from a Poisoned Chalice," Science 302 (October 17): 376-379.

Kuhn, Thomas (1970), The Structure of Scientific Revolutions. 2nd ed. Chicago: University of Chicago Press.

Lakatos, Imre (1970), "Falsification and the Methodology of Scientific Research Programmes," in Imre Lakatos and Alan Musgrave (eds.), Criticism and the Growth of Scientific Knowledge. Cambridge: Cambridge University Press, 91-196.

Laudan, Larry (1977), Progress and Its Problems. Berkeley: University of California Press.

Machamer, Peter, Lindley Darden, and Carl Craver (2000), "Thinking about Mechanisms," Philosophy of Science 67: 1-25.

Mayo, Deborah (1996), Error and the Growth of Experimental Knowledge. Chicago: University of Chicago Press.

Mayo, Deborah, and Aris Spanos (2006), "Philosophers of Science and Statistical Controversies in Ecological Testing," Philosophy of Science 73 (5), in this issue.

Nickles, Thomas (1997), "A Multi-pass Conception of Scientific Inquiry," Danish Yearbook of Philosophy 32: 11-44.

Popper, Karl (1959), The Logic of Scientific Discovery. New York: Basic Books.

Sarewitz, Daniel (2000), "Science and Environmental Policy: An Excess of Objectivity," in Robert Frodeman (ed.), Earth Matters: The Earth Sciences, Philosophy, and the Claims of Community. Upper Saddle River, NJ: Prentice-Hall, 79-98.

Schaffner, Kenneth (1993), Discovery and Explanation in Biology and Medicine. Chicago: University of Chicago Press.

Stebbing, A. R. D. (1998), "A Theory for Growth Hormesis," Mutation Research 403: 249 258.

Steinle, Friedrich (1997), "Entering New Fields: Exploratory Uses of Experimentation," Philosophy of Science 64 (supplement): S65-S74.

Vichi, P., and T. Tritton (1989), "Stimulation of Growth in Human and Murine Cells by Adriamycin," Cancer Research 49: 2679-2682.

Wimsatt, William (1987), "False Models as Means to Truer Theories," in Matthew Nitecki and Antoni Hoffman (eds.), Neutral Models in Biology. New York: Oxford University Press, 23-55.

Woodward, James (2000), "Data, Phenomena, and Reliability," Philosophy of Science 67: S163-S179. 